\title{
PRÁTICAS PEDAGÓGICAS INTERDISCIPLINARES COM O USO DE TEXTOS MULTIMODAIS
}

Mirtes Ribeiro de Lira (UPE)

Evandro Dias Amorim (UPE)

Resumo: A implementação de práticas pedagógicas interdisciplinares demanda a adoção de ações docentes inovadoras, capazes de promover a reorganização e ressignificação do conhecimento, como forma de adequar o ensino às reais necessidades da sociedade hodierna, e a multimodalidade por ser um fenômeno dinâmico, que congrega múltiplas linguagens e representações, torna-se um dos suportes imprescindíveis para a concretização destas ações. Este artigo apresenta um estudo sobre a produção científica nos mestrados profissionais em educação acerca de práticas pedagógicas interdisciplinares pautadas na utilização de textos multimodais. A pesquisa foi realizada no Banco de Dissertações dos Mestrados Profissionais em Educação em funcionamento no país e estendeu-se também aos seguintes periódicos: Revista Brasileira de Educação/ANPED, Educação e Pesquisa/USP e Educação e Realidade/ UFRGS. O estudo foi direcionado às produções desenvolvidas entre os anos de 2012 e 2016. Os resultados obtidos na pesquisa demostram que os estudos referentes às práticas pedagógicas interdisciplinares e à utilização de recursos linguísticos multimodais na prática docente são ainda muito incipientes, tanto nos mestrados profissionais em educação, quanto nos periódicos acima referenciados.

Palavras-chave: Práticas Interdisciplinares; Textos Multimodais; Mestrados Profissionais em Educação.

Abstract: The implementation of interdisciplinary pedagogical practices demands the adoption of innovative teaching actions, capable of promoting the reorganization and re-signification of knowledge, as a way of adapting teaching to the real needs of today's society, and multimodality as a dynamic phenomenon, that assembles multiple languages and representations, it becomes one of the indispensable supports for the accomplishment of these actions. This article presents a study about the scientific production in the education professional master about interdisciplinary pedagogical practices based on the use of multimodal texts. The research was carried out in the Bank of 
Dissertations of Education Professional Master in operation in the country and also extended to the following periodicals: Brazilian Journal of Education/ANPED, Education and Research/USP and Education and Reality/UFRGS. The study was directed to the productions developed between the years 2012 and 2016. The results obtained in the research show that the studies referring to interdisciplinary pedagogical practices and the use of multimodal linguistic resources in teaching practice are still very incipient, both in professional masters in education and in the periodicals mentioned above.

Keywords: Interdisciplinary Practices; Multimodal Texts; Professional Master in Education.

\section{INTRODUÇÃO}

O desenvolvimento de práticas de ensino inovadoras, voltadas para a produção e disseminação do conhecimento em sua totalidade e complexidade, tem sido um dos principais anseios da educação deste século e as práticas pedagógicas pautadas na interdisciplinaridade são uma das principais referências. Contudo, a implementação de novas práticas de ensino que possam ir além dos projetos coletivos demanda atitude, estudo e pesquisa sobre as relações existentes entre os diversos conteúdos disciplinares e temáticas abordadas em sala de aula, assim como sobre a realização de ações que favoreçam estas integrações.

A utilização de textos que circulam socialmente, caracterizados principalmente pela congregação de diversas linguagens e representações é outro aspecto relevante para a concretização dessas práticas. Para Fazenda (1993, p.108) 
é a partir da interagência de múltiplas linguagens que se constitui uma linguagem interdisciplinar e é por meio desta que se amplia a compreensão da realidade que se quer representar. O texto ainda é na atualidade um dos principais recursos utilizados nas práticas de ensino e, antes centrado na monomodalidade, tornou-se multimodal. Segundo Rojo (2015, p.108), o texto multimodal é aquele que é composto de mais de uma modalidade de linguagem ou sistema de signos, como as modalidades verbal, visual, gestual e sonora. O texto multimodal torna-se relevante em práticas pedagógicas interdisciplinares devido à sua capacidade de integrar conceitos e linguagens inerentes às distintas áreas do conhecimento.

Estudos científicos realizados acerca da realização de práticas pedagógicas interdisciplinares, apontam que as principais dificuldades enfrentadas pelos professores para a implementação e efetivação dessas práticas, estão relacionadas à ausência de uma formação específica e de propostas de ensino que possam nortear o trabalho docente (AUGUSTO, CALDEIRA, 2007; FAZENDA, 1979; FRIGOTTO, 1995; JAPIASSU, 2006).

A ampliação da oferta de programas de Mestrado Profissional em educação nos últimos anos tem sido 
um caminho viável para a superação desses obstáculos. Tais programas, além de representarem uma oportunidade ímpar de capacitação para os profissionais docentes, favorecem o desenvolvimento de propostas pedagógicas inovadoras, destinadas à promoção de uma educação de qualidade, em consonância com as transformações e demandas da sociedade contemporânea. De acordo com a Coordenação de Aperfeiçoamento de Pessoal de Nível Superior (CAPES, 2014), o produto final desenvolvido nos cursos de Mestrados Profissionais deve ter como objetivo a resolução de problemas reais, inerentes à área de atuação do profissionalmestrando.

Destarte, é de salutar importância um estudo aprofundado sobre as produções que estão sendo realizadas nos Mestrados Profissionais em educação em curso no país, bem como, sobre as publicações em destaque nos principais periódicos educacionais, principalmente as propostas de práticas pedagógicas interdisciplinares com a utilização de textos multimodais.

\section{MÉTODOS APLICADOS E RESULTADOS DO ESTUDO}

A investigação acerca da temática supramencionada concentrou-se especificamente nas publicações identificadas no Banco de Dissertações dos Mestrados Profissionais 
em educação em funcionamento no país, conforme lista fornecida pela Coordenação de Aperfeiçoamento de Pessoal de Nível Superior (CAPES). A opção por esta fonte de pesquisa deu-se em razão da preferência pela análise das produções realizadas por profissionais docentes, em sua maioria, em atuação na Educação Básica e pelas propostas direcionadas a este nível de ensino.

O estudo estendeu-se também à apreciação dos periódicos: Revista Brasileira de Educação/ANPED, Educação e Pesquisa/USP e Educação e Realidade/UFRGS. Estabeleceuse como parâmetro para a mencionada escolha, os estratos indicativos de qualidade estabelecidos pela Capes, neste caso específico o Qualis A1, e também a produção científica proveniente de distintas regiões do país. O estudo foi direcionado às produções desenvolvidas em um período de cinco anos, entre os anos de 2012 e 2016. Para a realização da busca nos mencionados periódicos e bancos de dissertações, foram escolhidos os termos "interdisciplinar" e "multimodal" e considerou-se também, outras palavras derivadas destes termos como: interdisciplinaridade e multimodalidade, constantes especificamente no título das produções.

Após uma pesquisa exaustiva em todos os segmentos, não foram identificadas produções que constassem no título 
os termos escolhidos. Diante do resultado insatisfatório, buscou-se empreender uma nova pesquisa com os mesmos termos, direcionando-a desta vez, às palavras-chave das referidas produções. Os números, bem como, a análise dos resultados obtidos neste estudo estão dispostos nos quadros a seguir.

\section{ANÁLISE DOS PERIÓDICOS IDENTIFICADOS NA PESQUISA}

\begin{tabular}{|l|c|c|c|c|c|c|}
\hline NÚMERO DE PUBLICAÇÕES POR PERIÓDICOS \\
\hline \multirow{2}{*}{ PERIÓDICOS } & 2012 & 2013 & 2014 & 2015 & 2016 & TOTAL \\
\cline { 2 - 8 } & 30 & 46 & 47 & 44 & 44 & 211 \\
\hline Revista Brasileira de Educação & 56 & 54 & 57 & 88 & 60 & 315 \\
\hline Educação e Pesquisa & 32 & 67 & 61 & 59 & 74 & 293 \\
\hline Educação e Realidade & \multicolumn{7}{|c|}{ ANOS } & 819 \\
\hline TOTAL GERAL
\end{tabular}

Quadro 1: Quadro demonstrativo do número de publicações por periódicos no período de 2012 a 2016

Conforme exposto no quadro acima, foram analisadas 819 (oitocentos e dezenove) publicações constantes dos periódicos selecionados, destas, 211 (duzentos e onze) são provenientes da Revista Brasileira de Educação, 315 (trezentos e quinze) da revista Educação e Pesquisa e 293 (duzentos e noventa e três) da Revista Educação e Realidade. A despeito do número considerável de produções analisadas, apenas dois artigos abordam a temática da interdisciplinaridade, ambos publicados na revista Educação e Pesquisa, no ano de 2015.

O primeiro artigo intitulado: “¿Se trabajan de forma interdisciplinar música y matemáticas en educación infantil?", 
de Rocío Chao Fernández; María Dorinda Mato Vázquez e Andrea Maria López Chao (2015), do supramencionado periódico, teve como objetivo investigar se os docentes da Educação Infantil trabalham matemática e música de forma interdisciplinar e como esse trabalho é desenvolvido. Nesse estudo as autoras apresentam diversas fontes bibliográficas que evidenciam as relações existentes entre as disciplinas de matemática e música, o que vem a corroborar para a proposição de um ensino interdisciplinar para as mencionadas disciplinas.

O mencionado artigo apresenta o resultado de uma pesquisa realizada com 360 professores de educação infantil e educação musical, pertencentes a escolas públicas e privadas da província de Coruña na Espanha. Por meio de um questionário misto, as autoras procuram identificar o modo como os professores trabalham as duas disciplinas; se a aula é ministrada pelo mesmo professor, se o planejamento é realizado de forma interdisciplinar ou por disciplinas.

Dentre os resultados apresentados por Chao Fernández et al. (2015), chamam a atenção as contradições identificadas nas respostas obtidas no questionário aplicado aos professores, uma vez que mais $80 \%$ dos entrevistados não se consideram suficientemente preparados para trabalhar matemática 
e música de forma interdisciplinar principalmente devido à falta de experiência no ensino de música, todavia, este mesmo percentual afirma avaliar essas duas disciplinas interdisciplinarmente. Segundo os autores, outro fator que dificulta o trabalho interdisciplinar das disciplinas em destaque, é a falta de empenho dos docentes. Para Chao Fernández et al, os docentes não demonstram interesse em buscar alternativas para a promoção de uma aprendizagem significativa, como a realização de projetos interdisciplinares e atividades extracurriculares.

Eles afirmam que os benefícios do ensino interdisciplinar de música e matemática são incontáveis, tendo em vista as diversas relações existentes entre essas disciplinas e classificam a formação docente abrangente, capaz de superar as barreiras disciplinares e também a renovação constante das metodologias de ensino e dos materiais e recursos empregados, como fatores primordiais para a promoção de uma aprendizagem significativa e uma educação integral.

O segundo artigo "Pensar arriscado: a relação entre filosofia e educação", de Nadja Hermann (2015), do mesmo periódico, aborda a inserção da filosofia no campo educacional e a sua relação com as demais ciências humanas, como a sociologia, a psicologia e a história, sob o prisma da 
interdisciplinaridade, cujo propósito é superar os limites da especialização e desenvolver um pensamento "capaz de produzir complexidades nas análises que iluminem as questões educacionais". O estudo é fruto de uma proposta de análise das relações existentes entre as disciplinas acima referenciadas para uma sessão especial da Associação Nacional de Pós-Graduação e Pesquisa em Educação (ANPEd), publicada no mês de outubro de 2013.

Nesse artigo, Hermann descreve a trajetória das ciências, e, em especial, da filosofia ao longo dos anos, principalmente no campo educacional. A autora ressalta que a filosofia não goza mais do status de "ciência primeira" e aponta a necessidade de diálogo entre as ciências, especialmente aquelas dedicadas ao ensino das humanidades na área da educação. Para Hermann (2015), esta nova concepção de filosofia da educação contribui para a produção de um "pensamento arriscado", ou seja, para o desenvolvimento da capacidade de produzir complexidades, quando se dispõe a indicar como um conceito pode ser utilizado para solucionar um problema em um determinado contexto.

Os artigos encontrados a partir da temática pesquisada, mesmo não tendo os textos multimodais como objeto de análise e também, não os relacionando às práticas 
pedagógicas interdisciplinares de modo explícito e intencional, mostram-se relevantes para este estudo. As proposições de trabalho apresentadas, além de apropriaremse da interdisciplinaridade para o ensino e aprendizagem de conteúdos, apropriam-se também da multimodalidade, como pode se constatar no artigo de Chao Fernández et al. (2015), uma vez que os elementos que constituem a música (letra e som), tornam-na por si só, um texto multimodal.

ANÁLISE DAS DISSERTAÇÕES IDENTIFICADAS NA PESQUISA

\begin{tabular}{|c|c|c|c|c|c|c|}
\hline \multicolumn{7}{|c|}{ NÚMERO DE DISSERTAÇÕES POR INSTITUIÇÃO DE ENSINO } \\
\hline \multirow[b]{2}{*}{ PERIÓDICOS } & \multicolumn{6}{|c|}{ ANOS } \\
\hline & 2012 & 2013 & 2014 & 2015 & 2016 & TOTAL \\
\hline $\begin{array}{l}\text { Centro Univ. Adventista de } \\
\text { São Paulo }\end{array}$ & - & - & - & - & - & - \\
\hline $\begin{array}{ll}\text { Centro } & \text { Universitário } \\
\text { Internacional } & \end{array}$ & - & - & 24 & 06 & - & 30 \\
\hline $\begin{array}{l}\text { Instituto Federal Sul-rio- } \\
\text { grandense }\end{array}$ & 03 & 09 & 20 & 19 & 22 & 73 \\
\hline $\begin{array}{l}\text { Pontifícia Universidade de } \\
\text { São Paulo }\end{array}$ & - & - & - & 23 & 41 & 64 \\
\hline $\begin{array}{l}\text { Universidade Alto Vale do } \\
\text { Rio do Peixe }\end{array}$ & - & - & - & - & - & - \\
\hline Universidade de Araraquara & - & - & - & 04 & 15 & 19 \\
\hline Universidade de Brasília & 01 & 05 & 23 & 32 & 29 & 90 \\
\hline $\begin{array}{l}\text { Universidade de } \\
\text { Pernambuco/Mata Norte }\end{array}$ & - & - & - & - & 07 & 07 \\
\hline $\begin{array}{ll}\text { Universidade } & \text { de } \\
\text { Pernambuco/Petrolina } & \\
\end{array}$ & - & - & - & - & 01 & 01 \\
\hline Universidade de Taubaté & - & - & - & - & - & - \\
\hline Universidade de Uberaba & - & - & - & - & - & - \\
\hline $\begin{array}{l}\text { Universidade Estadual da } \\
\text { Bahia }\end{array}$ & 37 & 42 & 26 & 30 & 21 & 156 \\
\hline
\end{tabular}




\begin{tabular}{|l|c|c|c|c|c|c|}
\hline $\begin{array}{l}\text { Universidade Estadual da } \\
\text { Paraíba }\end{array}$ & - & 01 & 25 & 15 & 03 & 44 \\
\hline $\begin{array}{l}\text { Universidade Estadual de } \\
\text { Mato Grosso do Sul }\end{array}$ & 01 & 14 & 23 & 20 & 09 & 67 \\
\hline $\begin{array}{l}\text { Universidade Federal da } \\
\text { Bahia }\end{array}$ & - & 06 & 44 & 56 & 15 & 121 \\
\hline $\begin{array}{l}\text { Universidade Federal da } \\
\text { Fronteira Sul }\end{array}$ & - & - & - & 19 & 20 & 39 \\
\hline $\begin{array}{l}\text { Universidade Federal de } \\
\text { Lavras }\end{array}$ & 20 & 20 & 20 & 20 & 24 & 104 \\
\hline $\begin{array}{l}\text { Universidade Federal de } \\
\text { Minas Gerais }\end{array}$ & - & - & - & 05 & 26 & 31 \\
\hline $\begin{array}{l}\text { Universidade Federal de } \\
\text { Rondônia }\end{array}$ & - & - & - & 13 & 14 & 27 \\
\hline $\begin{array}{l}\text { Universidade Federal de São } \\
\text { Carlos }\end{array}$ & - & - & 01 & 06 & 10 & 17 \\
\hline $\begin{array}{l}\text { Universidade Federal de } \\
\text { Uberlândia }\end{array}$ & - & - & 04 & 20 & 15 & 39 \\
\hline $\begin{array}{l}\text { Universidade Federal do } \\
\text { Pampa }\end{array}$ & - & - & 10 & 28 & 21 & 59 \\
\hline $\begin{array}{l}\text { Universidade Federal do } \\
\text { Paraná }\end{array}$ & - & - & - & 19 & 26 & 45 \\
\hline $\begin{array}{l}\text { Universidade Federal do } \\
\text { Tocantins }\end{array}$ & - & - & 10 & 09 & - & 19 \\
\hline $\begin{array}{l}\text { Universidade Federal dos } \\
\text { Vales do Jequitinhonha e } \\
\text { Mucuri }\end{array}$ & - & - & - & 12 & 10 & 22 \\
\hline $\begin{array}{l}\text { Universidade Municipal de } \\
\text { São Caetano do Sul }\end{array}$ & - & - & - & - & - & - \\
\hline $\begin{array}{l}\text { Universidade Nove de Julho } \\
\text { Total GERAL }\end{array}$ & - & 02 & 27 & 23 & 25 & 77 \\
\hline
\end{tabular}

Quadro 2: Quadro demonstrativo do número de dissertações por programas de Mestrado Profissional no período de 2012 a 2016

Conforme os dados acima mencionados, foram analisadas 1.151 (mil cento e cinquenta e uma) dissertações dos Programas de Mestrado Profissional aprovados pela Capes, em funcionamento no Brasil. Dos 27 (vinte e sete) Programas 
investigados, 05 (cinco) não apresentaram publicações, tendo em vista a recente implantação. A pesquisa direcionada às palavras-chave das mencionadas publicações identificou em 07 (sete) dissertações os termos escolhidos para investigação. As dissertações abaixo abordam a interdisciplinaridade e a multimodalidade em contextos distintos, mas coesos.

A primeira dissertação analisada, denominada: "Tecendo saberes para encontrar novos caminhos para ensinar Ciências: o percurso da Formação Continuada em Astronomia de professores da Escola Básica", produzida por Ana Paula Santos Pereira (2014), no Instituto Federal de Educação, Ciência e Tecnologia Sul-rio-grandense, buscou descrever o percurso e os resultados de uma formação continuada, direcionada a um grupo de professores da Educação Básica das redes pública e privada da cidade de São José do Norte, no Rio Grande do Sul. A referida formação é fruto de um projeto de extensão intitulado: "Astronomia na Escola: um olhar além de nossos jardins", desenvolvido pelo Instituto em questão, com o objetivo de promover a revisão e/ou construção de conceitos acerca da Astronomia, educação e aprendizagem, bem como, a utilização de equipamentos e softwares em sala de aula. No mencionado estudo, a autora defende a dinamização da formação continuada através de ações coletivas e colaborativas, como forma de concretizar as práticas pedagógicas interdisciplinares. 
A segunda dissertação pesquisada, intitulada "A formação de professores para os estudos do meio" foi produzida por José Roberto Goncalves Dias (2015) na Pontifícia Universidade de São Paulo. O estudo em questão foi realizado em uma escola de Educação Básica da cidade de São Paulo e teve como objetivo a elaboração de uma proposta de formação para professores que lecionam nos anos finais do Ensino Fundamental e também no Ensino Médio, para atuação em projetos de estudo do meio. Destaca-se na proposta, a concepção interdisciplinar de formação, que enfatiza a relevância da experiência profissional e do contexto educacional para a atuação docente. A metodologia empregada no estudo em destaque, estabeleceu a divisão da formação continuada em 15 encontros presenciais, distribuídos em 7 etapas de trabalho.

A etapa inicial correspondeu ao estudo aprofundado acerca do conceito de Estudo do Meio, interdisciplinaridade, aprendizagem e as condições necessárias para a aplicação do método em questão. Segundo o autor, o objetivo desta etapa preliminar foi fornecer subsídios teóricos, com a finalidade de fundamentar a ação a ser realizada.

A segunda etapa foi direcionada à parte prática da formação continuada, com ênfase na qualificação para atuação em 
projetos de Estudo do Meio, com vista ao aprimoramento do trabalho pedagógico e da aprendizagem. Dias (2015) fez uma síntese das etapas da formação e dos temas a serem trabalhados nos encontros presenciais, como Planejamento (Como planejar? Como intervir?), Reflexão (Reflexão sobre os resultados, os registros docentes), Transformação (Revisão das decisões), Avaliação (Instrumentos de avaliação) e Produtos (Projetos de Estudos do Meio e compartilhamento das ações desenvolvidas).

Para Dias (2015), o Estudo do Meio é uma prática pedagógica significativa e inovadora, capaz de promover novos processos de ensino e de aprendizagem, por meio da observação e interação entre professores e alunos e entre seus pares em espaços externos ao ambiente escolar. Na opinião do autor, as atividades de Estudo do Meio estimulam a curiosidade, aguçam o senso crítico e possibilitam o desenvolvimento de habilidades e competências tanto dos discentes quanto dos docentes e contribui significativamente para o fortalecimento das relações interpessoais. $\mathrm{O}$ autor ressalta que a realização de uma prática pedagógica assentada em Estudos do Meio demanda responsabilidade, dinamismo e entusiasmo e deve ser estimulada a despeito dos entraves enfrentados pelas instituições de ensino na atualidade. 
A terceira dissertação, denominada "Práticas corporais de aventura nas aulas de Educação Física: as possibilidades pedagógicas no 5ㅇ ano do Ensino Fundamental", produzida por Dilvano Leder de França (2016), no Programa de Mestrado Profissional da Universidade Federal do Paraná, apresenta o resultado de um estudo realizado com 30 alunos do 50 ano do Ensino Fundamental da Escola Municipal Professor Herley Mehl, localizada no bairro Pilarzinho, na cidade de Curitiba no Estado do Paraná.

A pesquisa em questão teve como objetivo analisar as possibilidades de vivências e experiências das Práticas Corporais nas aulas de Educação Física no contexto escolar, estabelecendo uma ação interdisciplinar entre Educação Física e Educação Ambiental, por meio de Práticas Corporais de Aventura nas modalidades de corrida de orientação, slackline, arvorismo, parkour e skate. Na dissertação, o autor buscou evidenciar a necessidade de se promover ações educativas que favoreçam a integração curricular e que sejam mais coerentes com o ideário ambientalista.

A quarta dissertação identificada na pesquisa, intitulada “A formação dos professores e o currículo do curso técnico em Agropecuária do IF - Sertão Pernambucano numa perspectiva de prática pedagógica interdisciplinar", 
produzida por Elisângela Campos Damasceno Sarmento (2016), no Mestrado Profissional em Formação de Professores e Práticas Interdisciplinares da Universidade de Pernambuco, propôs-se a analisar a estrutura curricular do Curso Técnico Integrado ao Médio em Agropecuária do Instituto Federal de Educação, Ciência e Tecnologia do Sertão Pernambucano IF-SERTÃO, localizado na cidade de Petrolina no Estado de Pernambuco.

O estudo foi direcionado aos professores das disciplinas de Biologia, Geografia, Caprinovinocultura e Solos II, com o objetivo de identificar as possibilidades de integração de diferentes áreas do conhecimento por meio de práticas interdisciplinares. O estudo buscou investigar também a formação dos professores e suas implicações para a realização de uma prática pedagógica interdisciplinar.

Na análise do Projeto Pedagógico Curricular, a autora aponta algumas discrepâncias relacionadas a determinadas nomenclaturas constantes do documento referenciado, bem como, relacionadas aos saberes disciplinares das Bases Comum e Técnica, visto que, embora sejam correlatos, encontram-se dispostos em anos letivos distintos. Sarmento sinaliza na pesquisa a necessidade de reestruturação e flexibilização do currículo, de planejamento coletivo 
e colaborativo e de implementação de políticas institucionais permanentes de formação de professores. Para a autora, as práticas interdisciplinares efetivas demandam uma ação integrada da tríade: formação de professores, currículo e prática pedagógica.

A última dissertação relacionada à temática da interdisciplinaridade, denominada "A física no Enem dos anos 2008 e 2009 sob o olhar do movimento: ciência, tecnologia e sociedade", produzida por Márcio Lima da Silva (2016) na Universidade Nove de Julho, teve como pretensão analisar o nível de interface dos itens relacionados à Física no Exame Nacional do Ensino Médio dos anos de 2008 e 2009, sob o prisma de uma educação em consonância com os ideais do Movimento Ciência, Tecnologia e Sociedade, no que diz respeito à promoção de uma abordagem e análise crítica dos conhecimentos produzidos pela ciência e pela tecnologia e suas possíveis implicações no meio social, assim como, as concepções de ensino fundamentadas na interdisciplinaridade e na contextualização, conforme indicação dos Parâmetros Curriculares Nacionais (2000).

Os resultados apresentados revelam, segundo o autor, uma evolução do referido exame no período analisado, tanto na estrutura, quanto nos conteúdos e abordagens. Na conclusão do estudo, Silva (2016) procurou enfatizar 
a relevância do Enem para a organização curricular, para a produção de materiais didáticos, assim como, para o delineamento da prática pedagógica.

As produções apresentadas a seguir compreendem as dissertações identificadas na pesquisa que abordam a temática da multimodalidade. A primeira dissertação, intitulada "O uso do Facebook no processo de ensinoaprendizagem de Língua Portuguesa", produzida por Flávia Raquel dos Santos Serafim (2014) na Universidade Estadual da Paraíba, teve como proposição a utilização do Facebook como uma ferramenta de interação e de suporte para o desenvolvimento de atividades sistemáticas de análise de gêneros, linguagem multimodal e mídias. O público alvo da pesquisa foi 32 estudantes do 2 o ano do Ensino Médio da Escola Estadual de Ensino Fundamental e Médio José Bronzeado Sobrinho, localizada na cidade de Remígio, no Estado da Paraíba.

No mencionado estudo, Serafim (2014) alerta para a necessidade de a escola apropriar-se dos recursos disponibilizados pelas novas tecnologias e utilizá-los no processo de ensino e aprendizagem de conteúdos, tendo em vista que as mudanças ocorridas nos modos de comunicação e interação sociais têm demandado novas formas de disseminação e apropriação do conhecimento 
e consequentemente, uma nova prática docente, capaz de agregar as múltiplas linguagens em evidência no meio social. Serafim conclui que a utilização dos recursos interacionais do Facebook, para a exploração dos textos dispostos em diversos gêneros textuais que circulam nessa rede social, exerce um papel importante no processo de ensino-aprendizagem.

A segunda dissertação identificada na pesquisa, denominada: "Blog: uma proposta para a prática de multiletramentos na escola", produzida por Maria Morganna da Silva Castro (2014) na Universidade Estadual da Paraíba, teve como objeto de estudo o uso das novas tecnologias como suporte pedagógico ao estudo do gênero e também a utilização de textos multimodais na prática pedagógica, sendo este último o que se relaciona diretamente com a temática investigada nesta pesquisa. A pesquisa foi realizada com 26 estudantes do 9ㅇ ano do Ensino Fundamental da Escola Suzete Dias Correia, localizada na zona urbana do município de Massaranduba na Paraíba. No estudo em questão, a autora defende a proposta de promoção do letramento digital, através de atividades virtuais de leitura e escrita socializadas em um Blog.

Na concepção da autora, o Blog é um espaço digital dinâmico que permite ao estudante realizar pesquisas, armazenar as informações coletadas e também compartilhá-las 
com os demais colaboradores. Castro (2014) comprovou em seu estudo que a utilização do Blog para a prática de leitura e interpretação de textos multimodais contribui para a promoção do multiletramento dos estudantes.

\section{CONSIDERAÇÕES FINAIS}

Os resultados da pesquisa aqui apresentados, denotam a relevância da temática investigada e expõem a carência de um aprofundamento dos estudos referentes à implantação de práticas pedagógicas interdisciplinares, principalmente nos Mestrados Profissionais em Educação. As produções destacadas neste estudo evidenciam, mesmo que de modo não intencional, uma relação de complementariedade e interdependência entre contextualização e interdisciplinaridade. Outro fator preponderante nas mencionadas produções é a indicação de uso das novas tecnologias como suporte à contextualização do ensino, tendo em vista o potencial que elas detêm de congregar recursos verbais, imagéticos e sonoros e de diversificar os modos de representação, tornando-as, deste modo, indispensáveis à educação contemporânea.

Finalmente, este estudo revela a inexistência de pesquisas e produções que tenham como objeto de estudo e proposição, a utilização de textos multimodais para a realização de práticas pedagógicas interdisciplinares. 
Os estudos referentes à multimodalidade mostraram-se muito incipientes no período investigado, tanto nos periódicos acima referenciados, quanto no banco de dissertações dos Mestrados Profissionais em Educação. No entanto, uma análise aprofundada das produções e proposições analisadas permite-nos inferir a existência de uma estreita relação entre linguagem virtual e multimodal e interdisciplinaridade.

\section{REFERÊNCIAS}

AUGUSTO, Thaís Gimenez da Silva; Ana Maria de Andrade CALDEIRA, (2007). "Dificuldades para implantação de práticas interdisciplinares em escolas estaduais, apontadas por professores da área de ciências da natureza". Investigações em Ensino de Ciências 12(1), 139-154. In https:// www.if.ufrgs.br/cref/ojs/index.php/ienci/article/view/481 Acesso em 10.Jun.2017.

BRASIL. Ministério da Educação. Coordenação de Aperfeiçoamento de Pessoal de Nível Superior - Capes (2014). Mestrado Profissional: o que é?. In http://capes.gov.br/avaliacao/sobre-a-avaliacao/mestradoprofissional-o-que-e Acesso em 17.Set.2017.

CASTRO, Maria Morganna da Silva (2014). Blog: uma proposta para a prática de multiletramentos na escola. 166f. (Dissertação - Programa de Pós-Graduação Profissional em Formação de Professores - PPGPFP) Universidade Estadual da Paraíba, Campina Grande.

CHAO FERNÁNDEZ, Rocío; MATO VÁZQUEZ, María Dorinda; LÓPEZ CHAO, Andrea Ma (2015). “¿Se trabajan de forma interdisciplinar música y matemáticas en educación infantil?" Educação e Pesquisa, 41(4), 10091022 Universidade de São Paulo São Paulo, Brasil.

DIAS, José Roberto Gonçalves (2015). A formação de professores para os estudos do meio. 69f. (Dissertação - Mestrado em Educação) - Pontifícia Universidade Católica de São Paulo, São Paulo. 
EDUCAÇÃO E PESQUISA (2012-2016). São Paulo: USP; 01-04. Quadrimestral .ISSN 1678-4634. In http://www.educacaoepesquisa.fe.usp.br/?cat=17 Acesso em 08.Jun.2017.

EDUCAÇÃO E REALIDADE (2012-2016). Porto Alegre: UFRGS; 01-04. Quadrimestral.ISSN 2175-6236. In http://seer.ufrgs.br/index.php/ educacaoerealidade/about Acesso em 07.Jul.2017.

FAZENDA, Ivani Catarina (1979). Integração e interdisciplinaridade no ensino brasileiro: Efetividade ou ideologia. São Paulo: Loyola.

(1993).(Org.). Práticas Interdisciplinares na Escola. São Paulo:

Cortez.

FRANÇA, Dilvano Leder de. (2016). Práticas corporais de aventura nas aulas de educação física: as possibilidades pedagógicas no 5o ano do ensino fundamental. (Dissertação) Curitiba: UFPR.

FRIGOTTO, Gaudêncio (1995). A interdisciplinaridade como necessidade e como problema nas ciências sociais. Petrópolis, RJ: Vozes.

HERMANN, Nadja (2015). "Pensar arriscado: a relação entre filosofia e educação". Educação e Pesquisa (USP. Impresso), 41, 217-228.

JAPIASSU, H. (2006). "O espírito interdisciplinar". Cadernos EBAPE FGV. 4(3) - Outubro, 1-9. In http://www.ebape.fgv.br/cadernosebape/arq/ Jupiassu.pdf Acesso em 17.Set.2017.

PEREIRA, Ana Paula Santos (2014). Tecendo saberes para encontrar novos caminhos para ensinar Ciências: o percurso da Formação Continuada em Astronomia de professores da escola básica. 230f. (Dissertação Mestrado Profissional em Educação e Tecnologia) - Instituto Federal Sulrio-grandense, Programa de Pós-Graduação em Educação, Pelotas.

REVISTA BRASILEIRA DE EDUCAÇÃO. Rio de Janeiro: ANPEd; n. 49-67, 2012-2016. Trimestral.ISSN: 1809-449X. In http://www.anped.org.br/site/ rbe/rbe. Acesso em 09.Mai.2017.

ROJO, R. H. R. (2013) Escola conectada: os multiletramentos e as TICS. São Paulo: Parábola. 
SARMENTO, Elisângela Campos Damasceno (2015). A formação dos professores e o currículo do curso técnico em Agropecuária do IF - Sertão Pernambucano numa perspectiva de prática pedagógica interdisciplinar. 144f. (Dissertação - Mestrado Profissional em Formação de Professores e Práticas Interdisciplinares) - Universidade de Pernambuco, Campus Petrolina.

SERAFIM, Flávia Raquel dos Santos (2014). O uso do facebook no processo de ensino-aprendizagem de língua portuguesa.112f. (Dissertação -Mestrado Profissional em Formação de Professores) - Universidade Estadual da Paraíba, Centro de Educação.

SILVA, Márcio Lima da (2016). A física no ENEM dos anos 2008 e 2009 sob o olhar do movimento: ciência, tecnologia e sociedade. 104f. (Dissertação Programa de Mestrado em Gestão e Práticas Educacionais) - Universidade Nove de Julho, São Paulo.

SOARES, Magda Becker; MACIEL, Francisca Pereira. Alfabetização no Brasil: o estado do conhecimento. In http://www.mec.inep.gov.br, 2000. Acesso em 12Abr.2017

Mirtes Ribeiro de Lira é Doutora em Educação pela Universidade Federal de Pernambuco. Professora adjunta da Universidade de Pernambuco, do Programa de Mestrado Profissional em Letras em Rede Nacional PROFLETRAS/UPE. E-mail: mirtes.lira@upe.br

Evandro Dias Amorim é Mestrando em Educação PPGFPPI - UPE. E-mail: evandrodamor@hotmail.com 